\title{
Identification of Treponema pedis as the predominant Treponema species in porcine skin ulcers by fluorescence in situ hybridization and high-throughput sequencing.
}

\author{
Karlsson, Frida; Schou, Kirstine Klitgaard; Jensen, Tim Kåre
}

Published in:

Veterinary Microbiology

Link to article, DOI:

10.1016/j.vetmic.2014.03.019

Publication date:

2014

Document Version

Publisher's PDF, also known as Version of record

Link back to DTU Orbit

Citation (APA):

Karlsson, F., Schou, K. K., \& Jensen, T. K. (2014). Identification of Treponema pedis as the predominant Treponema species in porcine skin ulcers by fluorescence in situ hybridization and high-throughput sequencing. Veterinary Microbiology, 171(1-2), 122-131. https://doi.org/10.1016/j.vetmic.2014.03.019

\section{General rights}

Copyright and moral rights for the publications made accessible in the public portal are retained by the authors and/or other copyright owners and it is a condition of accessing publications that users recognise and abide by the legal requirements associated with these rights.

- Users may download and print one copy of any publication from the public portal for the purpose of private study or research.

- You may not further distribute the material or use it for any profit-making activity or commercial gain

- You may freely distribute the URL identifying the publication in the public portal 


\title{
Identification of Treponema pedis as the predominant Treponema species in porcine skin ulcers by fluorescence in situ hybridization and high-throughput sequencing
}

\author{
Frida Karlsson ${ }^{\text {a,* }}$, Kirstine Klitgaard ${ }^{\text {b }}$, Tim Kåre Jensen ${ }^{\mathrm{b}}$ \\ ${ }^{a}$ Department of Clinical Sciences, Swedish University of Agricultural Sciences, SE-75007 Uppsala, Sweden \\ ${ }^{\mathrm{b}}$ National Veterinary Institute, Technical University of Denmark, 1870 Frederiksberg, Denmark
}

A R T I C L E I N F O

Article history:

Received 3 February 2014

Received in revised form 7 March 2014

Accepted 12 March 2014

\section{Keywords:}

Ear necrosis

FISH

Pig

Shoulder ulcer

Skin ulcer

Treponema

\begin{abstract}
A B S T R A C T
Skin lesions often seen in pig production are of great animal welfare concern. To study the potential role of Treponema bacteria in porcine skin ulcers, we investigated the presence and distribution of these organisms in decubital shoulder ulcers $(n=51)$ and ear necroses $(n=54)$ by fluorescence in situ hybridization (FISH) and high-throughput sequencing. In addition, two cases of facial ulcers and five cases of other skin ulcers were included in the study. Samples from all 112 skin lesions and intact skin from pigs without skin ulcers $(n=14)$ were screened by FISH. Three different oligonucleotide probes targeting $16 \mathrm{~S}$ rRNA were used, specific for domain bacterium, Treponema spp. and species T. pedis. Screening showed that two cases each of facial and other ulcers, 35 (69\%) of shoulder ulcers and 32 (59\%) of ear necroses were positive for Treponema spp. T. pedis was the unequivocally, predominant species typically constituting more than $90 \%$ of the treponemes in a lesion, assessed visually by microscopy. Altogether, T. pedis was demonstrated in 69 of the 71 Treponema spp. positive lesions. We conclude that Treponema spp. are frequently present and abundant in various skin ulcers of pigs. The results from this study point toward an important role of $T$. pedis as a secondary bacterial infection in porcine skin ulcers, especially in severe and chronic lesions.
\end{abstract}

(c) 2014 Elsevier B.V. All rights reserved.

\section{Introduction}

Skin ulcers of various kinds occur in pigs and have been intriguing scientists and veterinarians since the beginning of the last century. In different types of lesions, including generally spread skin ulcers, foot-rot, facial ulcers and ear necrosis, spirochetes have been identified and described, with some cases as early as 1906 (Blandford et al., 1972; Dodd, 1906; Harcourt, 1973; Hindmarsch, 1937; Osborne

\footnotetext{
* Corresponding author at: Department of Clinical Sciences, Swedish University of Agricultural Sciences (SLU), Box 7054, SE-750 07 Uppsala, Sweden. Tel.: +4618 671466; fax: +4618672919.

E-mail address: frida.karlsson@slu.se (F. Karlsson).
}

and Ensor, 1955). Transmission between animals was also reported (Dodd, 1906; Osborne and Ensor, 1955). In none of the studies mentioned above were any attempts to culture or further characterize the spirochetes described.

Bacteria of genus Treponema are involved in a number of diseases in the skin or mucus membranes in several mammals. In production animals, treponemes are associated with ulcerative mammary dermatitis (UMD) and bovine digital dermatitis (BDD) in cattle and contagious ovine digital dermatitis (CODD) in sheep (Naylor et al., 1998; Stamm et al., 2009; Walker et al., 1995). In BDD, isolates described as Treponema pedis, Treponema phagedenis-like, Treponema medium/Treponema vincentii-like and Treponema brennaborense have been obtained so far 
(Demirkan et al., 1999; Schrank et al., 1999; Stamm et al., 2002; Walker et al., 1995). The number of treponemal phylotypes identified by culture independent techniques is steadily increasing (Choi et al., 1997; Klitgaard et al., 2008, 2013; Rasmussen et al., 2012).

In 2008, spirochetes of species T. pedis were isolated and characterized from shoulder ulcers, ear necrosis and gingiva of pigs (Pringle et al., 2009; Pringle and Fellström, 2010). Recently, additional treponemal phylotypes occurring in these types of ulcers and in gingiva of pigs were detected (Karlsson et al., 2013). Two of three putative main phylotypes could be identified as species $T$. pedis and Treponema parvum (Svartström et al., 2013).

In this study fluorescence in situ hybridization (FISH) was used to detect microorganisms of the domain bacteria, genus Treponema and species T. pedis in 112 skin tissue samples from pigs with skin ulcers and in 14 skin tissue samples from pigs without lesions. In addition, lysates from 36 out of 112 skin lesions were investigated by high-throughput sequencing. Our aims were to study the occurrence, density and distribution of treponemes in biopsy sections from skin lesions in pigs, with emphasis on $T$. pedis and the gross and histopathological scoring of the lesions. We further sought to confirm the predominant occurrence of $T$. pedis and investigate the presence of other treponemes in a subset of samples by high-throughput sequencing.

\section{Materials and methods}

\subsection{Herds, animals and sampling procedure}

From April 2010 until December 2011, 105 animals with either decubital shoulder ulcers $(n=51)$ or ear necrosis $(n=54)$ were sampled. These animals belonged to eighteen Swedish pig herds (A-R). The samples, which consisted of both scrapings and biopsies, have been used in a previous study where herds, animals and sampling procedure were described (Karlsson et al., 2013). Of the lesions included in this study, 15 of the shoulder lesions had in the previous study been scored as mild and 36 as severe based on gross pathology. Likewise, the ear lesions had been scored as mild ( $n=26)$ and severe $(n=28)$. In the present study, two weaners from herd $L$ and three weaners from herd $\mathrm{R}$ were also sampled from other skin ulcers on the body, located to the neck or flank. In addition, two piglets with facial ulcers were sampled. These came from a herd with previously reported problems regarding ear necrosis (Pringle et al., 2009).

For comparison, punch biopsies were taken at an abattoir, from eight sows without shoulder ulcers and from six finisher pigs without ear lesions. These pigs originated from seven different herds and two pigs from each herd were sampled. The herds were not related to the herds in Karlsson et al. (2013). The biopsies were taken immediately after culling, but before scalding, from the skin of the shoulder region or the ventral margin of the ear.

\subsection{Preparation of samples}

The biopsies from all 126 animals were fixated in formalin and embedded in paraffin according to routine histological procedures. Sections $(\sim 5 \mu \mathrm{m})$ were cut and mounted on SuperFrost/plus slides (Menzel-Gläser, Braunschweig, Germany). Before hybridization paraffin was removed and the sections dehydrated by soaking the slides in xylene $(2 \times 2 \mathrm{~min}), 99 \%$ ethanol $(1 \times 2 \mathrm{~min})$ and $96 \%$ ethanol ( $1 \times 2 \mathrm{~min})$. Preparation of ulcer material for PCR analysis has previously been described (Karlsson et al., 2013).

\subsection{Oligonucleotide probes}

The oligonucleotide probes used in this study were directed toward $16 \mathrm{~S}$ rRNA. Probes had been designed previously and target the domain Bacteria (5'gctgcctccgtaggagt-3') (Amann et al., 1990), genus Treponema (5'-cagaaacycgccttcgcc-3'), covering known phylotypes occurring in BDD (Klitgaard et al., 2008), or T. pedis (5'-agagtcctcaacctttacgtgtt-3') (Rasmussen et al., 2012). Furthermore, a probe for $T$. parvum was designed (5'ccactggcttcgggtatcct $3^{\prime}$ ), using methods described previously (Klitgaard et al., 2008). Both the T. pedis and the $T$. parvum probes had more than three mismatches to the negative controls applied in this study. The oligonucleotide probe for domain Bacteria was $5^{\prime}$ labeled with fluorescein isothiocyanate (FITC), and the probes for Treponema spp., $T$. pedis and T. parvum with the isothiocyanate derivative $\mathrm{Cy} 3$ (Eurofins MWG Operon, Ebersberg, Germany, or DNA Technology A/S, Risskov, Denmark).

\subsection{Hybridization}

The hybridization was performed at $46^{\circ} \mathrm{C}$ with hybridization buffer ( $100 \mathrm{mM}$ Tris [pH 7.2], $0.9 \mathrm{M} \mathrm{NaCl}$ $0.1 \%$ sodium dodecyl sulfate) and $5 \mathrm{ng} / \mu \mathrm{L}$ of each probe for $16 \mathrm{~h}$ in a Sequenza slide rack (Thermo Shandon, Cheshire, United Kingdom). After hybridization the sections were washed three times $(3 \times 3 \mathrm{~min})$ in prewarmed $\left(46^{\circ} \mathrm{C}\right)$ hybridization buffer and similarly in prewarmed $\left(46^{\circ} \mathrm{C}\right)$ washing solution ( $100 \mathrm{mM}$ Tris [pH 7.2], $0.9 \mathrm{M} \mathrm{NaCl}$ ). The sections were rinsed in MilliQ water, air dried and mounted with Vectashield (Vector Laboratories Inc. Burlingame, CA).

\subsection{Epifluorescence microscopy}

An axioimager M1 epifluorescence microscope with a 120-W HBO lamp and filter sets 43 and 38 were used to visualize Cy3 and FITC, respectively, and double filter 24 to visualize both green and red fluorescence. Photos were taken using an AxioCam MRm version 3 FireWire monochrome camera and AxioVision software, version 4.5 (Carl Zeiss, Oberkochen, Germany).

\subsection{Specificity of probes}

One isolate each of $T$. pedis (TA4) (Pringle et al., 2009), $T$. parvum (B1119) (Svartström et al., 2013) and T. phagedenislike (V1) (Pringle et al., 2008) were cultured for 3-6 days to obtain a dense culture (Svartström et al., 2013). Each culture was centrifuged to a pellet and material from the pellets was injected into small tissue samples of healthy 
porcine lung and fixated in formalin. Sections were prepared, hybridized with the probe for either $T$. pedis or T. parvum, and investigated by microscopy as described above.

\subsection{Screening for Treponema spp. and Treponema pedis}

Each section was double hybridized with the domain bacteria probe (FITC, green) and the general Treponema spp. probe ( $\mathrm{Cy} 3$, red), screened for presence of treponemes and given a score of 0-3 similar to the scoring system described by Rasmussen et al. (2012) in biopsies from BDD, 0 (no treponemes were detected), 1 (the amount of bacteria detected with the probe was sparse, $<5 \%$ of the total amount of bacteria visualized in the section), 2 (the amount of bacteria detected was moderate, $5-10 \%$ of total) or 3 (the amount of bacteria detected was abundant, $>10 \%$ of total). The area with the highest density of Treponema spp. was chosen for the scoring. Scoring was based on visual assessment. Serial sections of all Treponema spp. positive sections were furthermore double hybridized with the probe for T. pedis and domain Bacteria and scored as above.

\subsection{Histopathological evaluation}

After epifluorescence microscopy the cover glasses were removed and the slides were stained by hematoxylin and eosin (HE) for histopathological evaluation. A varying degree of ulceration bordered by acanthotic epithelium had previously been described (Karlsson et al., 2013) thus, this evaluation focused on characterizing the severity of the lesions. The degree of epidermal/dermal damage in the lesion was scored from 0 to 3 . Score 0 was defined as normal epidermis/dermis, score 1 as focal erosion/ulceration, score 2 as widespread ulceration with presence only of solitary epidermal pegs in dermis, score 3 as diffuse ulceration. Furthermore, lesions without plasma cells and granulation tissue were graded as acute whereas lesions infiltrated by plasma cells and granulation tissue were graded as chronic.

\subsection{Selection of samples for $16 S$ rRNA PCR}

A limited number of lysates from scrapings of skin lesions were selected for $16 S$ rRNA PCR. Preparation of lysates from ulcer scraping material has previously been described (Karlsson et al., 2013). The selection criterion was that the lysate had been positive for Treponema spp. by intergenic spacer region 2 (ISR2)-based PCR (Karlsson et al., 2013) or that the corresponding biopsy had been positive for Treponema spp. by FISH (this study). The selected samples represented various types of skin ulcers (32 shoulder ulcers, 23 ear necroses, two facial ulcers and two other ulcers) and were from 59 individual pigs. The pigs originated from 17 out of 19 visited herds.

\subsection{Preparation of $16 \mathrm{~S}$ rRNA gene amplicon libraries and sequencing}

For sequencing, lysates from skin lesion scrapings were amplified by PCR using Treponema-specific primers targeting the V3 and V4 hypervariable regions, which spanned positions 346-705 in Escherichia coli K-12. Each sample was amplified with unique forward and reverse primers that included an added hexamer barcode at their 5 ' ends (Klitgaard et al., 2013). The DNA concentration and quality of the PCR amplicons were assessed with an Agilent 2100 bioanalyzer (Agilent Technologies Inc., Santa Clara, CA) prior to high-throughput sequencing (data not shown). Equal amounts of all amplicons were pooled (final amount of $2.4 \mu \mathrm{g}$ ) and purified using the Qiagen Mini Elute kit (Qiagen; Hilden, Germany), according to the protocol provided by the manufacturer.

The DNA was submitted to the National High-throughput DNA Sequencing Center at Copenhagen University, Denmark, for sequencing on an Illumina MiSeq ${ }^{\mathrm{TM}}$ platform. The obtained 226-bp long reads were analyzed using the BION-meta software (http://box.com/bion). De-multiplexing was performed according to the primer- and barcode sequences. Next, the sequences were cleaned at both ends by removal of bases of a quality less than $98 \%$, which is equivalent to a Phred score of 17 . Identical sequences were clustered into consensus sequences. Consensus sequences of at least 190 nucleotides in length were mapped into a table according to the individual barcodes. Finally, the consensus sequences were taxonomically classified against the Ribosomal Database Project database (RDP; http://rdp.cme.msu.edu/index.jsp) using a word length of 8 and a match minimum of $97 \%$. To allow comparison of the relative abundance between samples, the number of reads for each barcode was normalized. The resulting microbial profiles were further analyzed in Microsoft's Excel. The data discussed in this publication have been deposited in the NCBI Sequence Read Archive (http:// www.ncbi.nlm.nih.gov/Traces/sra/) and are accessible through accession number: SRP036648.

\section{Results}

\subsection{Specificity of FISH probes}

Investigation by fluorescent microscopy resulted in a bright detectable signal only from the sections containing $T$. pedis, hybridized with the probe for $T$. pedis. No fluorescent signal was detected from the sections containing $T$. phagedenis-like or T. parvum when hybridized with the $T$. pedis probe. The probe for $T$. parvum generated a signal too weak to be satisfactory interpreted and was excluded from the study.

\subsection{Screening for Treponema spp. and T. pedis by FISH}

The results from screening with the genus probe are shown in Table 1 . Spirochetes of genus Treponema were detected in 71 (63\%) of the biopsies from ulcers. Separating the different kinds of ulcers, $69 \%$ of the shoulder ulcers and $59 \%$ of the ear necroses were positive for treponemes. Of all shoulder ulcers, $49 \%$ had a score of 3 . The corresponding percentage for ear necroses was 22\%. Furthermore, bacteria of genus Treponema were confirmed in both cases of facial ulcers (score 3 ) and in two out of five cases of other ulcers (score 2 and 3, respectively). None of the sections 
Table 1

Screening of Treponema spp. and Treponema pedis by fluorescence in situ hybridization in biopsies from various skin ulcers $(n=112)$ and intact skin $(n=14)$ of pigs.

\begin{tabular}{|c|c|c|c|c|c|}
\hline Treponema spp./T. pedis scoring ${ }^{\mathrm{a}}$ & 0 & 3 & 2 & 1 & Total $(n)$ \\
\hline \multicolumn{6}{|l|}{ Location of biopsy } \\
\hline Shoulder ulcer & 16 & $25^{\mathrm{b}}$ & 5 & $5^{\mathrm{d}}$ & 51 \\
\hline Ear necrosis & 22 & $12^{\mathrm{b}}$ & $10^{\mathrm{c}}$ & $10^{\mathrm{d}}$ & 54 \\
\hline Facial ulcers & - & $2^{c}$ & - & - & 2 \\
\hline Other ulcers & 3 & 1 & 1 & - & 5 \\
\hline All ulcers & 41 & 40 & 16 & 15 & 112 \\
\hline Shoulder skin (no ulcer) & 8 & - & - & - & 8 \\
\hline Ear skin (no ulcer) & 6 & - & - & - & 6 \\
\hline
\end{tabular}

a Scoring system according to Rasmussen et al. (2012): 0 - no hybridization of treponemes; 1 - the amount of bacteria detected with the probe was sparse, $<5 \%$ of the total amount of bacteria visualized in the section; 2 - the amount of bacteria detected was moderate, $5-10 \%$ of total or $3-$ the amount of bacteria detected was abundant, $>10 \%$ of total.

b In one biopsy $T$. pedis was recorded as score 2

c In one biopsy $T$. pedis was recorded as score 1 .

d One biopsy was negative for $T$. pedis.

from the 14 animals without ulcers were positive for treponemes.

Additional hybridization of the Treponema positive biopsies for $T$. pedis showed a very similar pattern revealing 69 biopsies positive. Furthermore, the scoring results from the $T$. pedis screening corresponded well with the results from the Treponema spp. screening, with only six cases differing from the original scoring result (Table 1). Four of these six cases were still scored as positive for $T$. pedis (score 1-2). A section from a shoulder ulcer with a score of 3 , and an ear necrosis with a score of 2 , double hybridized with the probe for T. pedis and domain bacteria, are shown in Figs. 1a and 2a.

\subsection{Treponemal occurrence according to gross lesions and histopathological evaluation}

Histopathologically, the 14 biopsies of healthy skin were within normal range except for a few small pustules, focal epidermal hyperkeratosis, and mild perivascular dermatitis. Of the lesions only 13 biopsies (12\%), including 11 ears, one shoulder, and one other ulcer, showed no evident plasma cell proliferation nor granulation tissue (and were considered as acute lesions) while $88 \%$ of the lesions showed varying degree of chronic ulceration with plasma cells and formation of granulation tissue. However, many of the chronic ear lesions also showed foci with more acute changes. There were sections positive for treponemes among both acute and chronic lesions.

Of the grossly mild shoulder lesions $47 \%$ were positive for Treponema spp. compared to $78 \%$ of the grossly severe lesions. In both mild and severe shoulder lesions the lowest Treponema occurrence was found in the lesions characterized by focal ulceration only. Of the grossly mild ear lesions $58 \%$ were positive for Treponema spp. compared to $61 \%$ of the grossly severe ones. In the ear lesions the pattern of treponemal occurrence varied more than in the shoulder ulcers. The distribution of Treponema scoring according to the gross and histopathological scoring of the lesions is shown in Tables 2 and 3 for shoulder and ear biopsies, respectively.

In shoulder ulcers, a Treponema score of 3 was the most common score, representing $71 \%$ of the positive samples.
Typically, in lesions with Treponema score 3 the Treponema organisms were densely packed side by side vertically to the surface forming a broad brim on the border between vital granulation tissue and surface debris as shown in Fig. 1a and b. Due to the severity of the lesions, epidermis was missing in many samples. Extensive necrosis and formation of granulation tissue made it difficult to define if the treponemes were located to epidermis, dermis or the epidermal-dermal border. In few cases Treponema organisms were found within degenerated, acanthotic epithelium but never on the epithelial surface. Bacteria other than treponemes, mostly cocci, were also observed but usually found closer to the surface than the treponemes. A smaller proportion of treponemes were found in the granulation tissue below the brim decreasing in number with increasing depth, Fig. 1c. In some cases treponemes were found beyond $0.5 \mathrm{~mm}$ in the granulation tissue which easily exceeded $5 \mathrm{~mm}$ in thickness. The treponemes invading the granulation tissue were found in the intercellular space showing no tropism for specific cells and were only associated with a low leukocytic response (Fig. 1d). However, in some cases the treponemes were found clustering around blood vessels. Interestingly, the presence of treponemes deep in the granulation tissue did not influence the leukocyte response compared to Treponema negative lesions. The surfaces of the Treponema negative lesions were generally colonized by a mixed bacterial biofilm of varying density and thickness.

In contrast to the results obtained with the shoulder biopsies, in which the individual lesions appeared rather uniform regarding age and severity, many of the ear biopsies showed foci of varying age and epithelial damage within the lesion. Six lesions showed no ulceration but diffuse exudative epidermitis. Of these six, two were Treponema positive (score 2). Lesions with treponemes forming a thick brim similar to that seen in shoulders lesions with Treponema score 3 were observed among the ear lesions, but were not as common. Even so, Treponema score 3 was the most frequent score. An example of an ear lesion is shown in Fig. 2a. This lesion was given Treponema score 2 . The treponemes and other bacteria were found infiltrating superficially the acanthotic and degenerated epithelium found widespread in the lesion. The HE stained 


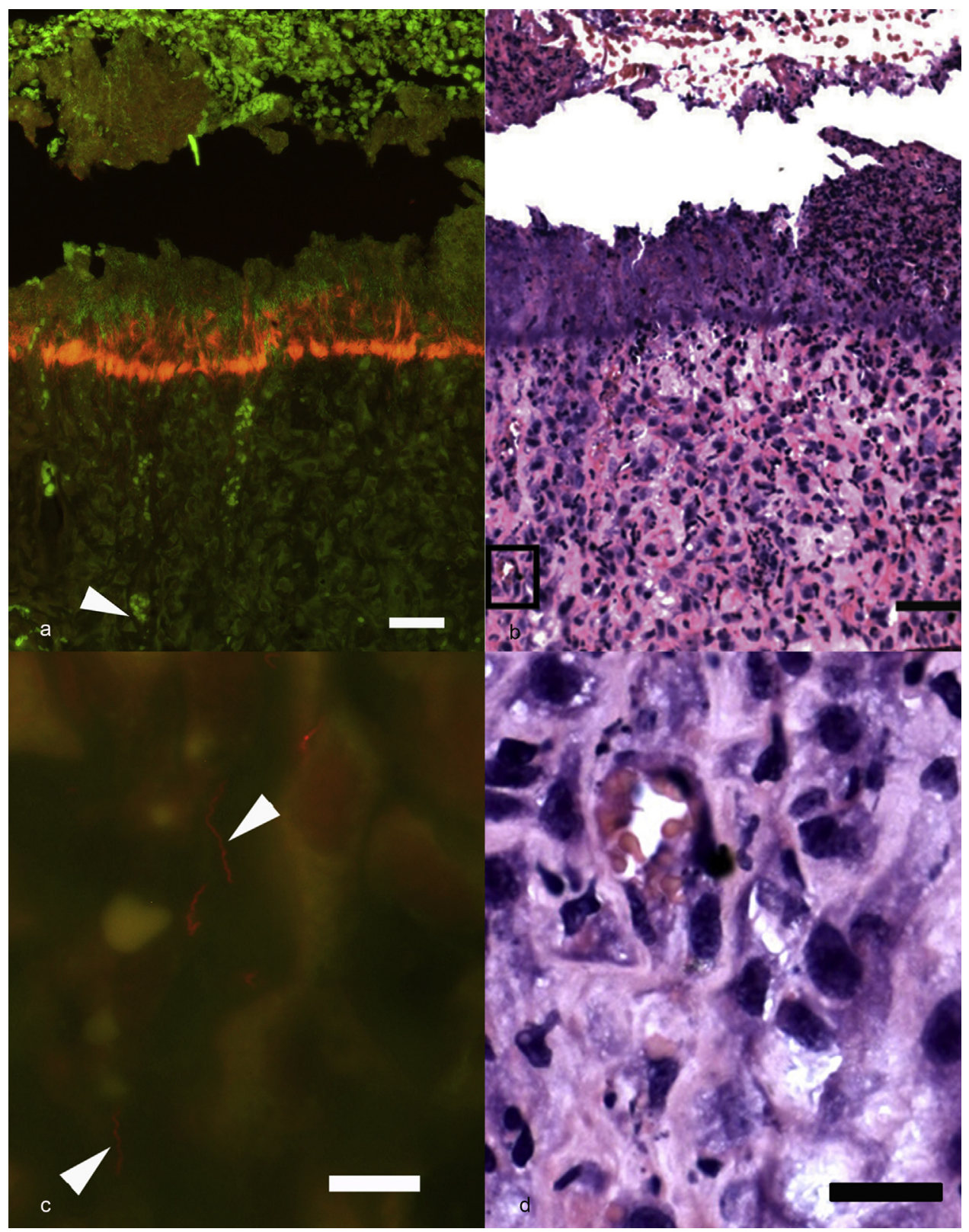

Fig. 1. Biopsy of shoulder ulcer from one sow. (a) Treponema pedis (orange/red) in high number (score 3 ) forming a thick brim on the border between granulation tissue and debris covering the ulcer. Other bacteria are green. Please note that due to autofluorescence erythrocytes are visible as bright green spheres. One example is the green mass located close to the arrowhead. Below the brim, T. pedis in low numbers infiltrate the granulation tissue deep down (arrowhead). Fluorescence in situ hybridization for T. pedis (CY3) and domain bacteria (FITC), bar $100 \mu \mathrm{m}$. (b) Same section as $1 \mathrm{a}$ after staining by HE visualizing the thick granulation tissue of the ulcer. The leukocytic response is mild in the superficial layers despite the presence of $T$. pedis. Bar $25 \mu \mathrm{m}$. (c) Close up of the area around the arrowhead/black box in 1a and $1 \mathrm{~b}$ demonstrating single T. pedis organisms (red) deep in the granulation tissue, bar $10 \mu \mathrm{m}$. (d) Same section as 1c after staining by HE revealing granulation tissue and almost total absence of leukocytes despite $T$. pedis infiltration, bar $25 \mu \mathrm{m}$. (For interpretation of the references to color in this legend, the reader is referred to the web version of the article.)

Fig. $2 \mathrm{~b}$ represents the same lesion at a lower magnification with the area in Fig. 2a in the black box. In many ear lesions other bacteria than treponemes were found in large clusters as shown in Fig. 2c.

Comparing the density and distribution between parallel sections hybridized with the probes for Treponema spp. or T. pedis, it was evident that $T$. pedis was far the most common species. In fact it was only possible to clearly detect other treponemes in very few lesions and only in areas with relative low treponemal density, as shown in Fig. $2 \mathrm{~d}$. Several of the biopsies from ulcers with relative high occurrence of $T$. medium/T. vincentii-like treponemes, as shown by high-throughput sequencing, were carefully examined by FISH without finding strong evidence of other treponemes than $T$. pedis. It should be noted, however, that in areas with high density of $T$. pedis their bright 


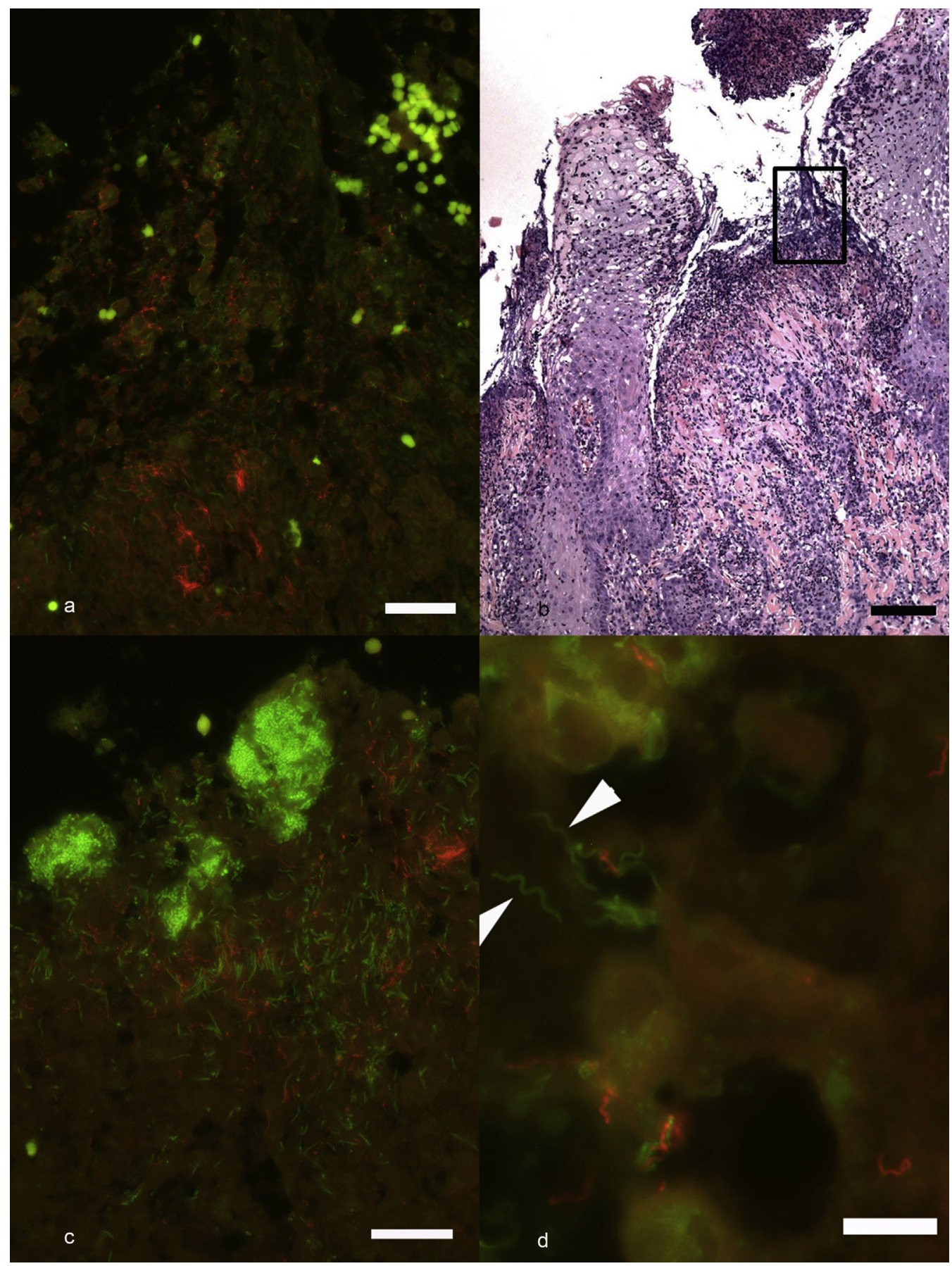

Fig. 2. Biopsies of ear necrosis from three pigs. (a) Treponema pedis (red) (score 2) and other bacteria (green) superficially infiltrating necrotic tissue covering a focal ulceration. Fluorescent in situ hybridization for T. pedis (CY3) and domain bacteria (FITC), bar $25 \mu \mathrm{m}$. (b) Same section as 2a after staining by HE but at a lower magnification, showing the focal ulcer surrounded by degenerated and necrotic epidermis. Bar $150 \mu \mathrm{m}$. (c) Demonstration of bacteria (green) other than treponemes (red) forming large clusters in degenerated epidermis, bar $25 \mu \mathrm{m}$. The figure is not from the same animal as $2 \mathrm{a}$ and $2 \mathrm{~b}$. (d) Demonstration of T. pedis (red) and other treponemes (spirochetes) (green) close together in degenerated epidermis, bar $10 \mu \mathrm{m}$. The figure is not from the same animal as $2 \mathrm{a}, 2 \mathrm{~b}$ or $2 \mathrm{c}$. (For interpretation of the references to color in this legend, the reader is referred to the web version of the article.) 
Table 2

Shoulder ulcers in pigs. Gross lesions, histopathology scoring and detection of Treponema spp. by fluorescence in situ hybridization.

\begin{tabular}{|c|c|c|c|c|c|c|}
\hline \multirow[t]{2}{*}{ Histopathology } & \multirow{2}{*}{$\begin{array}{l}T \text {. spp. negative } \\
\text { Score } 0\end{array}$} & \multicolumn{3}{|c|}{ T. spp. positive } & \multirow[t]{2}{*}{ T. spp. positive total } & \multirow[t]{2}{*}{ Total } \\
\hline & & Score 3 & Score 2 & Score 1 & & \\
\hline \multicolumn{7}{|l|}{ Gross lesion mild shoulder ulcers } \\
\hline Ulceration, no epithelium (score 3 ) & 2 & 3 & 1 & 2 & 6 & $8(53 \%)$ \\
\hline Ulceration but focal epithelial pegs in dermis (score 2) & 5 & 0 & 0 & 0 & 0 & $5(33 \%)$ \\
\hline Focal ulceration only (score 1 ) & 1 & 1 & 0 & 0 & 1 & $2(13 \%)$ \\
\hline Total & $8(53 \%)$ & 4 & 1 & 2 & $7(47 \%)$ & 15 \\
\hline \multicolumn{7}{|l|}{ Gross lesion severe shoulder ulcers } \\
\hline Ulceration, no epithelium (score 3 ) & 4 & 14 & 2 & 1 & 17 & $21(58 \%)$ \\
\hline Ulceration but focal epithelial pegs in dermis (score 2 ) & 3 & 6 & 1 & 2 & 9 & $12(33 \%)$ \\
\hline Focal ulceration only (score 1 ) & 1 & 1 & 1 & 0 & 2 & $3(8 \%)$ \\
\hline Total & $8(22 \%)$ & 21 & 4 & 3 & $28(78 \%)$ & 36 \\
\hline
\end{tabular}

Table 3

Ear necroses in pigs. Gross lesions, histopathology scoring and detection of Treponema spp. by fluorescence in situ hybridization.

\begin{tabular}{|c|c|c|c|c|c|c|}
\hline \multirow[t]{2}{*}{ Histopathology } & \multirow{2}{*}{$\begin{array}{l}T \text {. spp. negative } \\
\text { Score } 0\end{array}$} & \multicolumn{3}{|c|}{ T. spp. positive } & \multirow[t]{2}{*}{ T. spp. positive total } & \multirow[t]{2}{*}{ Total } \\
\hline & & Score 3 & Score 2 & Score 1 & & \\
\hline \multicolumn{7}{|l|}{ Gross lesion mild ear necrosis } \\
\hline Ulceration, no epithelium (score 3 ) & 1 & 1 & 0 & 1 & 2 & $3(12 \%)$ \\
\hline Ulceration but focal epithelial pegs in dermis (score 2 ) & 6 & 3 & 3 & 0 & 6 & $12(46 \%)$ \\
\hline Focal ulceration only (score 1 ) & 1 & 3 & 1 & 1 & 5 & $6(23 \%)$ \\
\hline Exudative epidermitis, no ulceration (score $1 / 2$ ) & 3 & 0 & 2 & 0 & 2 & $5(19 \%)$ \\
\hline Total & $11(42 \%)$ & 7 & 6 & 2 & $15(58 \%)$ & 26 \\
\hline \multicolumn{7}{|l|}{ Gross lesion severe ear necrosis } \\
\hline Ulceration, no epithelium (score 3) & 7 & 4 & 2 & 3 & 9 & $16(57 \%)$ \\
\hline Ulceration but focal epithelial pegs in dermis (score 2 ) & 2 & 0 & 1 & 3 & 4 & $6(21 \%)$ \\
\hline Focal ulceration only (score 1 ) & 1 & 1 & 1 & 2 & 4 & $5(18 \%)$ \\
\hline Exudative epidermitis, no ulceration (score $1 / 2$ ) & 1 & 0 & 0 & 0 & 0 & $1(4 \%)$ \\
\hline Total & $11(39 \%)$ & 5 & 4 & 8 & 17 (61\%) & 28 \\
\hline
\end{tabular}

hybridization signal interfered with the signals from the pan-bacterial probe making it difficult to clearly observe individual bacteria including treponemes.

\subsection{Sequence analysis}

Using oligonucleotide primers, designed to target the V3 and V4 hypervariable regions of 16S rRNA sequences of T. putidum, T. pedis, T. denticola, T. vincentii, T. calligyrum, and $T$. refringens, we were able to successfully PCR amplify and sequence 16S rRNA fragments of treponemes from 26 shoulder ulcers and 10 ear ulcers. Of the 59 samples that were submitted to PCR, 23 did not give positive bands. The sequence files from the Illumina $\mathrm{MiSeq}^{\mathrm{TM}}$ System were analyzed using the Bion-Meta software. A total number of $15,170,120$ reads were obtained from the sequencing center. After de-multiplexing according to the sequences of barcodes and primers $12,715,326$ sequences were left and these were further $3^{\prime}$ and $5^{\prime}$ trimmed according to the quality. Sequences below a quality of $98 \%$ were discarded.

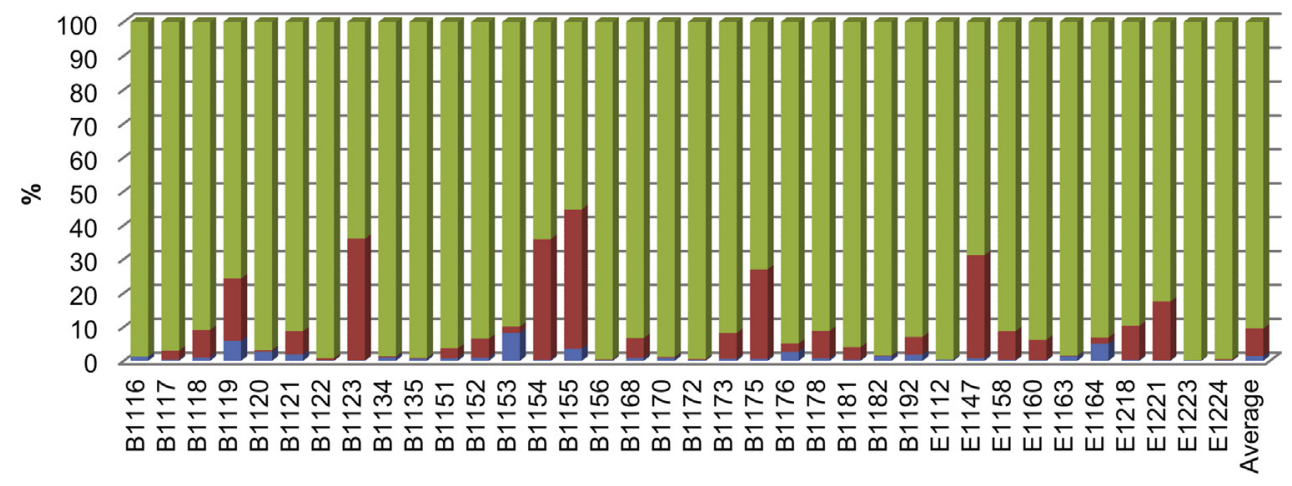

- Treponema spp. $\quad \square$. medium/T. vincentii-like $\square T$. pedis

Fig. 3. Relative frequencies of occurrence of the Treponema species in 36 porcine skin samples, identified by high-throughput sequencing of V3 and V4 hypervariable regions of $16 \mathrm{~S}$ rRNA. 
The number of sequences used for taxonomical classification was then $5,362,633$, which was equivalent to an average of 141,121 reads per sample. Of these, $54.5 \%$ were taxonomically classifiable according to the RDP database.

The sequences could be delineated into three groups. On average, $90.5 \%$ of the sequences were homologous to $T$. pedis, $8.2 \%$ mapped to an uncultured Treponema clone, 90q_2, which, in a BLAST search (http://blast.ncbi.nlm.nih.gov/Blast.cgi), displayed $98 \%$ sequence homology to $T$. medium and $T$. vincentii (henceforward referred to as $T$. medium/T. vincentii-like). The third group, here named Treponema spp., contained the remaining phylotypes which all had an average frequency well below $0.5 \%$. Included in this group were classified species such as $T$. denticola, T. berlinense, T. porcinum and T. amylovorum as well as an array of not-yet cultivated oral treponemes from dogs. The relative frequencies of occurrence of the three treponemal groups in the individual samples are shown in Fig. 3.

\section{Discussion}

In this study FISH was used to visualize bacteria of genus Treponema and species $T$. pedis in 112 skin tissue samples from ear necrosis, shoulder ulcers, facial ulcers and other ulcers of pigs. Sections from pigs with intact skin were also investigated $(n=14)$. In addition, the relative frequencies of various Treponema species were investigated in a subset of 36 samples by high-throughput sequencing. This is, to the best of our knowledge, the first study to detect treponemes in porcine skin ulcers by FISH or massive parallel sequencing and also the first published identification of spirochetes in facial and other ulcers of pigs as Treponema spp.

FISH has been used before to detect treponemes in samples from BDD (Choi et al., 1997; Klitgaard et al., 2008, 2013; Moter et al., 1998; Nordhoff et al., 2008; Rasmussen et al., 2012). The treponemal probes used in this study had been designed in two of the aforementioned studies (Klitgaard et al., 2008; Rasmussen et al., 2012) for use on bovine skin tissue and worked well also on porcine skin tissue.

In this study, the occurrence of Treponema spp. detected by FISH was 69\% in shoulder ulcers and 59\% in ear necroses, compared to the original study by Karlsson et al. (2013) where treponemes were detected in $52 \%$ of the shoulder ulcers and in $46 \%$ of the ear necroses by an ISR2-based PCR. It is however not possible to compare these results fully. The material used in each method does indeed originate from the same animal and ulcer, but for the ISR2-based PCR, lysates on superficial scraping material was used, whereas for FISH the material consisted of sections from full thickness biopsies. It is also possible that the lower detection rate by ISR2-based PCR was caused by a high level of porcine DNA or other inhibiting contents in the scraping sample.

The histopathological findings of both ear necroses and shoulder ulcers were in agreement with earlier descriptions of similar lesions (Jensen, 2009; Richardson et al., 1984). The main part of the investigated skin lesions represented varying degrees of chronic ulceration. Only a very small part (13 biopsies) was classified as acute. Even though treponemes could be confirmed also in acute lesions, the findings of this study must be regarded with focus on chronic ulcers. Within the shoulder lesions the histopathological changes appeared uniform in age and severity, whereas among the ear lesions the variation of the changes was greater. Six ear lesions showed signs of diffuse exudative epidermitis. Of these, only two were Treponema positive. The signs of exudative epidermitis are in agreement with the hypothesis of Staphylococcus hyicus as an initiator of ear necrosis (Mirt, 1999; Park et al., 2013; Richardson et al., 1984). In this study the presence of $S$. hyicus was not investigated.

With regard to the pathogenesis of decubital shoulder ulcers in sows, compression of the blood vessels in the skin around the tuber spina scapulae leads to circulation disturbances initiating thrombosis in dermis and necrosis of epidermis. The result is sloughing of epidermis, ulceration and secondary infection (Herskin et al., 2011; Jensen, 2009). In the present study cases representing the initial stage of decubital lesions were not found as all cases showed ulceration.

The treponemes were mainly found colonizing the border between debris and granulation tissue in the ulcer. In the present study, bacteria other than Treponema spp. were mostly limited to the debris covering the ulcers, with a sparse occurrence in sections with abundance of treponemes. The treponemes were not found on top of the ulcer, but within the lesion. This is also in agreement with previously published results from investigations of skin lesions in BDD in cattle (Klitgaard et al., 2008; Moter et al., 1998; Nordhoff et al., 2008; Rasmussen et al., 2012).

In this study, we chose to specifically investigate the occurrence of $T$. pedis in the ulcers using FISH. This was mainly due to previous findings suggesting $T$. pedis as a main phylotype occurring in porcine shoulder ulcers and ear necroses (Karlsson et al., 2013). Furthermore, T. pedis is frequently mentioned as one of several treponemal species associated with BDD, together with $T$. phagedenis-like and T. medium/T. vincentii-like (Brandt et al., 2011; Choi et al., 1997; Evans et al., 2009; Klitgaard et al., 2013; Nordhoff et al., 2008; Yano et al., 2010). T. phagedenis-like spirochetes were not found in porcine skin ulcers in the study by Karlsson et al. (2013) and T. medium was only sparsely detected, but frequent occurrence of $T$. parvum was indicated (Karlsson et al., 2013; Svartström et al., 2013). Unfortunately, due to difficulties in designing a specific probe, were not able to investigate the occurrence of $T$. parvum by FISH. We did not find any unique region situated in an area of the 16S rRNA where the fluorescent signal could be detected. To our knowledge, T. parvum has not been found in BDD, but has been isolated from human periodontitis (Wyss et al., 2001) and a porcine shoulder ulcer (Svartström et al., 2013).

The scoring results using either the Treponema genus probe or the specific probe for $T$. pedis were similar, as well as the appearance of the fluorescence pattern in the sections, which indicate that $T$. pedis constituted the main part of the detected treponemes in the investigated ulcers. In the study by Karlsson et al. (2013) several phylotypes of treponemes were detected in ear necrosis and shoulder 
ulcers in pigs by an ISR2-based PCR, followed by sequencing. To investigate the presence of other Treponema spp. in the lesions in this study we PCR amplified and sequenced a variable region of the 16S rRNA gene from a subset of porcine skin samples. The primers applied targets all the Treponema species hitherto identified in BDD skin specimens (Klitgaard et al., 2013). Of the 59 samples that were submitted to PCR, 23 failed to give positive bands. This may be explained by the quality of template DNA. The ulcers had been sampled in a previous study (Karlsson et al., 2013), and the lysates had therefore been stored in the freezer for more than a year. It should be emphasized that the lysates originated from scraping material and not from the biopsy material investigated by FISH, but in each case from the same ulcer. The dominance of $T$. pedis found with both techniques therefore shows that the treponemes were detected not only in sections of full thickness biopsies but also in more superficial scraping material.

The sequence analysis results supported the observations made by using FISH. In the 36 samples investigated by high-throughput sequencing, $T$. pedis on average accounted for $90.5 \%$ of the species identified. T. pedis was highly abundant in all samples (between $55.4 \%$ and 99.8\%) (Fig. 3). The second most prevalent species was a $T$. medium $/ T$. vincentii-like phylotype which on average constituted $8.2 \%$ of the sequences. In 13 of the skin samples, however, this phylotype was absent or had a frequency below $1 \%$. Species with high prevalence in BDD, e.g. T. denticola and T. phagedenis (Klitgaard et al., 2013) displayed extremely low abundance in all the samples investigated. This is in agreement with the previous study (Karlsson et al., 2013). It is therefore possible that $T$. medium/T. vincentii, $T$. denticola and T. phagedenis are of less importance to skin lesions in pigs.

Although the primers used to amplify treponemal rDNA in this investigation cover a broad array of members from the $T$. pallidum, $T$. phagedenis, and $T$. denticola subgroups (Klitgaard et al., 2013), they are not designed to amplify $T$. parvum. Consequently, we were not able to estimate frequency and prevalence of this species, and possibly other species of Treponema. It further needs to be determined to what extent other phylotypes occur in the ulcers, and how they are distributed in the tissue. An investigation using general bacterial primers or broader Treponema primers in combination with additional oligonucleotide probes targeting more treponemal species could be used in future studies of pig skin ulcers. In BDD, consensus among researchers is pointing toward a polytreponemal etiology. The issue on treponemal infections as part of the etiology of porcine skin ulcers, especially chronic ones, still needs further attention.

\section{Conclusion}

In conclusion, Treponema spp. are frequently present and abundant in shoulder ulcers and ear necroses in pigs. They also occur in facial ulcers and other ulcers on the body. The results point toward an important role of $T$. pedis as a secondary bacterial infection in porcine skin ulcers, especially in chronic and severe ones, as this species constituted a major part of the bacteria in the samples from the investigated ulcers.

\section{Acknowledgements}

We would like to thank Ewa Westergren, Annie Ravn Pedersen, Anastasia Isbrand and Joanna Zeitman Amenuvor for excellent technical assistance. We are also grateful to Märit Pringle and Annette Backhans for valuable comments. The project was financed by The Swedish Research Council Formas.

\section{References}

Amann, R.I., Binder, B.J., Olson, R.J., Chisholm, S.W., Devereux, R., Stahl, D.A., 1990. Combination of $16 S$ rRNA-targeted oligonucleotide probes with flow cytometry for analyzing mixed microbial populations. Appl. Environ. Microb. 56, 1919-1925.

Blandford, T.B., Bygrave, A.C., Harding, J.D., Little, T.W., 1972. Suspected porcine ulcerative spirochaetosis in England. Vet. Rec. 90, 15.

Brandt, S., Apprich, V., Hackl, V., Tober, R., Danzer, M., Kainzbauer, C., Gabriel, C., Stanek, C., Kofler, J., 2011. Prevalence of bovine papillomavirus and Treponema DNA in bovine digital dermatitis lesions. Vet. Microbiol. 148, 161-167.

Choi, B.K., Nattermann, H., Grund, S., Haider, W., Gobel, U.B., 1997. Spirochetes from digital dermatitis lesions in cattle are closely related to treponemes associated with human periodontitis. Int. J. Syst. Bacteriol. 47, 175-181.

Demirkan, I., Carter, S.D., Hart, C.A., Woodward, M.J., 1999. Isolation and cultivation of a spirochaete from bovine digital dermatitis. Vet. Rec. 145, 497-498.

Dodd, S., 1906. A disease of the pig, due to a spirochaeta. J. Comp. Pathol. Teurapeut. 19, 216-222.

Evans, N.J., Brown, J.M., Demirkan, I., Murray, R.D., Birtles, R.J., Hart, C.A., Carter, S.D., 2009. Treponema pedis sp nov., a spirochaete isolated from bovine digital dermatitis lesions. Int. J. Syst. Evol. Microbiol. 59, $987-$ 991.

Harcourt, R.A., 1973. Porcine ulcerative spirochaetosis. Vet. Rec. 92, 647648.

Herskin, M.S., Bonde, M.K., Jørgensen, E., Jensen, K.H., 2011. Decubital shoulder ulcers in sows: a review of classification, pain and welfare consequences. Animal 5, 757-766.

Hindmarsch, W.L., 1937. Ulcerative granuloma of pigs (spirochaetal tumours of pigs). New South Wales Department of Agriculture. Vet. Res. Rep. 7, 64-70.

Jensen, H.E., 2009. Investigation into the pathology of shoulder ulcerations in sows. Vet. Rec. 165, 171-174.

Karlsson, F., Svartström, O., Belák, K., Fellström, C., Pringle, M., 2013. Occurrence of Treponema spp. in porcine skin ulcers and gingiva. Vet. Microbiol. 165, 402-409.

Klitgaard, K., Boye, M., Capion, N., Jensen, T.K., 2008. Evidence of multiple Treponema phylotypes involved in bovine digital dermatitis as shown by $16 \mathrm{~S}$ rRNA gene analysis and fluorescence in situ hybridization. J. Clin. Microbiol. 46, 3012-3020.

Klitgaard, K., Breto, A.F., Boye, M., Jensen, T.K., 2013. Targeting the treponemal microbiome of digital dermatitis infections by high-resolution phylogenetic analyses and comparison with fluorescent in situ hybridization. J. Clin. Microbiol. 51, 2212-2219.

Mirt, D., 1999. Lesions of so-called flank biting and necrotic ear syndrome in pigs. Vet. Rec. 144, 92-96.

Moter, A., Leist, G., Rudolph, R., Schrank, K., Choi, B.K., Wagner, M., Göbel, U.B., 1998. Fluorescence in situ hybridization shows spatial distribution of as yet uncultured treponemes in biopsies from digital dermatitis lesions. Microbiology 144 (Pt 9) 2459-2467.

Naylor, R.D., Martin, P.K., Jones, J.R., Burnell, M.C., 1998. Isolation of spirochaetes from an incident of severe virulent ovine footrot. Vet. Rec. 143, 690-691.

Nordhoff, M., Moter, A., Schrank, K., Wieler, L.H., 2008. High prevalence of treponemes in bovine digital dermatitis - a molecular epidemiology. Vet. Microbiol. 131, 293-300.

Osborne, H.G., Ensor, C.R., 1955. Some aspects of the pathology, aetiology, and therapeutics of foot-rot in pigs. N. Z. Vet. J. 3, 91-99.

Park, J., Friendship, R.M., Poljak, Z., DeLay, J., Slavic, D., Dewey, C.E., 2013. An investigation of ear necrosis in pigs. Can. Vet. J. Rev. Vet. Can. 54, 491-495. 
Pringle, M., Fellström, C., 2010. Treponema pedis isolated from a sow shoulder ulcer. Vet. Microbiol. 142, 461-463.

Pringle, M., Bergsten, C., Fernström, L.L., Höök, H., Johansson, K.E., 2008. Isolation and characterization of Treponema phagedenis-like spirochetes from digital dermatitis lesions in Swedish dairy cattle. Acta Vet. Scand. 50, 40.

Pringle, M., Backhans, A., Otman, F., Sjölund, M., Fellström, C., 2009. Isolation of spirochetes of genus Treponema from pigs with ear necrosis. Vet. Microbiol. 139, 279-283.

Rasmussen, M., Capion, N., Klitgaard, K., Rogdo, T., Fjeldaas, T., Boye, M., Jensen, T.K., 2012. Bovine digital dermatitis: possible pathogenic consortium consisting of Dichelobacter nodosus and multiple Treponema species. Vet. Microbiol. 160, 151-161.

Richardson, J.A., Morter, R.L., Rebar, A.H., Olander, H.J., 1984. Lesions of porcine necrotic ear syndrome. Vet. Pathol. 21, 152-157.

Schrank, K., Choi, B.K., Grund, S., Moter, A., Heuner, K., Nattermann, H., Gobel, U.B., 1999. Treponema brennaborense sp. nov., a novel spirochaete isolated from a dairy cow suffering from digital dermatitis. Int. J. Syst. Bacteriol. 49 (Pt 1) 43-50.

Stamm, L.V., Bergen, H.L., Walker, R.L., 2002. Molecular typing of papillomatous digital dermatitis-associated Treponema isolates based on analysis of 16S-23S ribosomal DNA intergenic spacer regions. J. Clin. Microbiol. 40, 3463-3469.

Stamm, L.V., Walker, R.L., Read, D.H., 2009. Genetic diversity of bovine ulcerative mammary dermatitis-associated Treponema. Vet. Microbiol. 136, 192-196.

Svartström, O., Karlsson, F., Fellström, C., Pringle, M., 2013. Characterization of Treponema spp. isolates from pigs with ear necrosis and shoulder ulcers. Vet. Microbiol. 166, 617-623.

Walker, R.L., Read, D.H., Loretz, K.J., Nordhausen, R.W., 1995. Spirochetes isolated from dairy cattle with papillomatous digital dermatitis and interdigital dermatitis. Vet. Microbiol. 47, 343-355.

Wyss, C., Dewhirst, F.E., Gmur, R., Thurnheer, T., Xue, Y., Schupbach, P., Guggenheim, B., Paster, B.J., 2001. Treponema parvum sp nov., a small, glucuronic or galacturonic acid-dependent oral spirochaete from lesions of human periodontitis and acute necrotizing ulcerative gingivitis. Int. J. Syst. Evol. Microbiol. 51, 955-962.

Yano, T., Moe, K.K., Yamazaki, K., Ooka, T., Hayashi, T., Misawa, N., 2010. Identification of candidate pathogens of papillomatous digital dermatitis in dairy cattle from quantitative $16 \mathrm{~S}$ rRNA clonal analysis. Vet. Microbiol. 143, 352-362. 